\title{
FOUNDATIONS OF BQO THEORY
}

\author{
ALBERTO MARCONE
}

\begin{abstract}
In this paper we study the notion of better-quasi-ordering (bqo) originally defined by Nash-Williams [14]. In particular we consider the approximation to this concept given by the notion of $\alpha$-wqo, for $\alpha$ a countable indecomposable ordinal [15]. We prove that if a quasi-ordering $Q$ is $\alpha$-wqo then $Q^{<a}$ is wqo, thereby obtaining a new proof of Nash-Williams' theorem that $Q$ bqo implies $\tilde{Q}$ (the set of all countable transfinite sequences of elements of $Q$ ) bqo. We show that for $\alpha<\alpha^{\prime}, \alpha^{\prime}$-wqo is properly stronger than $\alpha$-wqo. We also show that the definition of $\alpha$-wqo (and therefore also of bqo) can be modified by considering only barriers with a nice additional property. In the last part of the paper we establish a conjecture of Clote [3] by proving that the set of indices for recursive bqos is complete $\Pi_{2}^{1}$.
\end{abstract}

The most natural generalization of the notion of well-ordering to partial orderings is the concept of well-quasi-ordering or wqo. A binary relation is a quasi-ordering if it is reflexive and transitive (it is a partial ordering if it is also antisymmetric) and a quasi-ordering is wqo if it contains no infinite descending sequences and no infinite antichains (sets of mutually incomparable elements). Ramsey's theorem implies that a quasi-ordering $Q$ is wqo if and only if for any function $f: \mathbb{N} \rightarrow Q$ there exist $n<m$ such that $f(n) \leq_{Q} f(m)$. For a history of the notion of wqo see [5]; for a survey of wqo (and bqo) theory see [10] and [16].

Unfortunately the notion of wqo does not enjoy nice closure properties: when we apply an operation in some sense "infinitary" to a wqo we cannot expect the resulting quasi-ordering to be wqo. This shortcoming prompted Nash-Williams, in a sequence of papers culminating in [14], to introduce the notion of betterquasi-ordering or bqo, i.e., well-quasi-ordering of a particularly well-behaved kind. Since then a vast theory of bqos has been developed and has provided the tools for proving that many natural quasi-orderings are bqo, and a fortiori wqo. One of the most important results in this area is Laver's theorem [6] establishing Fraïsse's conjecture that the class of countable linear orders is wqo

Received by the editors February 11, 1993.

1991 Mathematics Subject Classification. Primary 06A07.

Key words and phrases. Well-quasi-ordering, better-quasi-ordering, transfinite sequences, barrier, $\Pi_{2}^{1}$-completeness.

This research has been partially supported by a research assistantship from NSF Grant DMS 9002072.

This paper is part of the author's Ph.D. dissertation at The Pennsylvania State University. I wish to thank my advisor Professor Simpson, who introduced me to bqo theory and has encouraged me throughout this research, and Professor Pouzet, who invited me to Lyon and explained to me his theorem (Theorem 1.8 in this paper), which eventually led to the results of $\S \S 3$ and 5 . 
under embeddability. In recent years the original definition of bqo formulated by Nash-Williams, which has a strong combinatorial flavor, has been substituted by an equivalent definition, formulated originally by Simpson in [22], which uses Borel maps and allows the use of many powerful tools from descriptive set theory in bqo theory. This new approach has spurred a new wealth of results, e.g., see [7]. In this paper we will use Nash-Williams' original definition because it lends itself nicely to the fine analysis of the notion of bqo we are pursuing.

In [15] Pouzet proved the basic result that $Q$ is bqo if and only if $\tilde{Q}$, the set of all countable transfinite sequences of elements of $Q$ quasi-ordered by embeddability, is wqo (indeed the need for $\tilde{Q}$ to be wqo is what led NashWilliams to the definition of bqo). In the same paper Pouzet defined the notion of $\alpha$-wqo for a countable ordinal $\alpha$. This notion, which is most interesting when $\alpha$ is an indecomposable ordinal (i.e., $\alpha=\omega^{\beta}$ for some $\beta$ ), is a natural approximation of the notion of bqo: $Q$ is bqo if and only if it is $\alpha$-wqo for all countable $\alpha$. Moreover $\omega$-wqo coincides with wqo. Section 1 contains the definition of $\alpha$-wqo and some basic technical results which will be used in the following sections.

Reference [15] contains a proof that if $Q^{<\alpha}$ (the subset of $\tilde{Q}$ consisting of sequences of length less than $\alpha$ ) is wqo then $Q$ is $\alpha$-wqo. The converse of this result was stated by Pouzet but no proof of it ever appeared: we will prove it as Theorem 2.8. The proof of Theorem 2.8 leads to a proof of Nash-Williams' theorem "if $Q$ is bqo then $\tilde{Q}$ is bqo" which is more effective than the usual ones which make use of the so-called minimal bad array lemma. In particular our proof can be formalized in the subsystem of second order arithmetic $\Pi_{1}^{1}-\mathbf{C A}_{0}$ (see [9] for details on this formalization), while the classical proof appears to require the stronger system $\Pi_{2}^{1}-\mathbf{C A}_{0}$. Shore [21] has proved that Nash-Williams' theorem (even restricted to the special case where $Q$ is a singleton) implies (over the weak base system $\mathbf{R C A}_{0}$ ) the (properly weaker than $\Pi_{1}^{1}-\mathbf{C A}_{0}$ ) system $\mathbf{A T R}_{\mathbf{0}}$ and we conjecture that our proof can indeed be carried out in $\mathbf{A T R}$, thereby establishing the exact logical strength of this basic result of bqo theory. Some partial results towards establishing this conjecture are contained in [9]. Reference [3] contains a proof in $\mathbf{A T R}_{\mathbf{0}}$ of Nash-Williams' theorem but unfortunately that proof is incorrect, as Clote himself has acknowledged (personal communication). For general background on the various subsystems of second order arithmetic mentioned above see, e.g., [23].

The examples given by Pouzet to show that the notions of $\alpha$-wqo and $\alpha^{\prime}$-wqo are distinct when $\alpha \neq \alpha^{\prime}$ are indecomposable, are unfortunately incorrect, and we replace them in $\S 3$ : it appears that finding an explicit and uniform definition (analogous to the one originally proposed by Pouzet) for these examples is hopeless, and thus we resort to a different technique, which is based on a new result obtained by Pouzet [17] that links quasi-ordering to arbitrary binary relations and is proved in $\S 1$. The results of $\S \S 2$ and 3 together show that $Q$ bqo is not equivalent to $Q^{<\alpha}$ wqo for any countable $\alpha$.

In $\S 4$ we give an alternative but equivalent definition of the notion of $\alpha$-wqo by substituting Nash-Williams' barriers with the smooth barriers defined in $\S 3$, which have some nice additional properties. Obviously this substitution leads also to an alternative definition of the notion of bqo that could simplify the proofs of some results of bqo theory. 
In $\S 5$ we show that the notion of bqo is indeed more complex than that of wqo by proving that the set of indices for recursive bqos is complete $\Pi_{2}^{1}$ (the corresponding set for wqo is clearly $\Pi_{1}^{1}$ ). This was conjectured by Clote in [3]. It is possible that this set will play for $\Pi_{2}^{1}$ a role analogous to that played by the set of indices for recursive well-orderings for $\Pi_{1}^{1}$, namely that to prove that a set is complete $\Pi_{2}^{1}$ it will be natural to reduce to it the set of recursive bqos. Similar results are obtained for the set of all bqos on $\mathbf{N}$ (viewed as a subset of the Cantor space $2^{\omega}$ ). The proof uses some of the techniques introduced in $\S \S 1,3$, and 4 and in particular Pouzet's theorem and the concept of smooth barrier.

\section{BASIC DEFINITIONS AND TECHNICAL RESULTS}

Notation. If $s$ is a finite sequence (i.e., a function with domain a natural number) we denote by $\operatorname{lh}(s)$ its length and, for every $i<\operatorname{lh}(s)$, by $s(i)$ its $(i+1)$ th element. Then we write this sequence as $s=\langle s(0), \ldots, s(\operatorname{lh}(s)-1)\rangle$. If $s$ and $t$ are finite sequences we write $s \sqsubseteq t$ if $s$ is an initial segment of $t$, i.e., if $\operatorname{lh}(s) \leq \operatorname{lh}(t)$ and $\forall i<\operatorname{lh}(s) s(i)=t(i)$. We write $s \subseteq t$ if the range of $s$ is a subset of the range of $t$, i.e., if $\forall i<\operatorname{lh}(s) \exists j<\operatorname{lh}(t) s(i)=t(j)$. $s[t$ and $s \subset t$ have the obvious meanings and we extend these notations also to the case where $t$ is an infinite sequence (i.e., a function with domain $\mathbf{N}$ ).

We write $s^{\imath} t$ for the concatenation of $s$ and $t$, i.e., the sequence $u$ such that $\ln (u)=\operatorname{lh}(s)+\operatorname{lh}(t)$, for every $i<\operatorname{lh}(s) u(i)=s(i)$ and for every $i<\operatorname{lh}(t)$ $u(\operatorname{lh}(s)+i)=t(i)$.

If $s$ is a finite sequence and $i \leq \operatorname{lh}(s)$ we denote by $s[i]$ the initial segment of $s$ of length $i$, i.e., the unique sequence $t$ such that $t \subseteq s$ and $\operatorname{lh}(t)=i$. If $\operatorname{lh}(s)>0$ we denote by,$s$ the sequence obtained from $s$ by deleting its first element, i.e., ${ }_{*} s=\langle s(1), \ldots, s(\operatorname{lh}(s)-1)\rangle$. These notations are extended to infinite sequences as well. $2^{<\omega}$ (resp. $2^{\omega}$ ) denotes the set of all finite (resp. infinite) sequences of 0 's and 1 's. Similarly $\omega^{<\omega}$ and $\omega^{\omega}$ denote the sets of sequences of elements of $\mathbb{N}$.

If $X \subseteq N$ we denote as usual by $[X]^{<\omega}$ (resp. $[X]^{\omega}$ ) the set of all finite (resp. infinite) subsets of $X$. Throughout this paper we will identify a subset of $\mathbb{N}$ with the unique sequence enumerating it in increasing order. Therefore if $s \in[N]^{<\omega}$ we have that $\operatorname{lh}(s)$ is the cardinality of $s$ and, for $i<\operatorname{lh}(s), s(i)$ is the $(i+1)$ th least element of $s$. Similarly if $X \in[\mathbb{N}]^{\omega}$ then $X(i)$ is the $(i+1)$ th least element of $X$.

If $n \in \mathbb{N}$ we write $2^{n}$ (resp. $\omega^{n}$ ) for the set of all sequences of $2^{<\omega}$ (resp. $\left.\omega^{<\omega}\right)$ of length $n$ and $[X]^{n}$ for the set of all elements of $[X]^{<\omega}$ of cardinality $n$.

Definition. If $B$ is a subset of $[\mathbb{N}]^{<\omega}$ we denote by base $(B)$ the set

$$
\{n \mid \exists s \in B \exists i<\operatorname{lh}(s) s(i)=n\} .
$$

The following definitions are basic for bqo theory and were first given by Nash-Williams in [13] and [14], where the notion of better quasi-ordering was introduced.

Definition. A set $B \subseteq[\mathbb{N}]^{<\omega}$ is a block if it satisfies the following conditions:

(1) base $(B)$ is infinite; 
(2) $\forall X \in[\operatorname{base}(B)]^{\omega} \exists s \in B s \subset X$;

(3) $\forall s, t \in B s \not \subset t$.

$B$ is a barrier if it satisfies (1), (2), and

(3)' $\forall s, t \in B s \not \subset t$.

Since $s \subset t$ implies $s \subset t$ it is immediate that every barrier is a block. Notice that if $B$ is a block and $Y \in[\text { base }(B)]^{\omega}$ there exists a unique block $B^{\prime} \subseteq B$ such that base $\left(B^{\prime}\right)=Y$, namely $B^{\prime}=\{s \in B \mid s \subset Y\}$. Notice also that if $B$ is a barrier then $B^{\prime}$ is also a barrier and we say that $B^{\prime}$ is a subbarrier of $B$.

The following results are essentially due to Nash-Williams [12]. Reference [2] is an in-depth analysis of these theorems and contains full proofs of them.

Theorem 1.1.

(1) If $B$ is a block and $B=B_{1} \cup B_{2}$ then there exists $a$ block $B^{\prime}$ such that either $B^{\prime} \subseteq B_{1}$ or $B^{\prime} \subseteq B_{2}$.

(2) If $B$ is a barrier and $B=B_{1} \cup B_{2}$ then there exists a barrier $B^{\prime}$ such that either $B^{\prime} \subseteq B_{1}$ or $B^{\prime} \subseteq B_{2}$.

Proof. (1) is essentially the so-called clopen Ramsey theorem, see, e.g., [22, Theorem 9.6] or $[4,3.2 .3]$. (2) follows from (1) because any block contained in a barrier is a barrier.

Lemma 1.2 [13]. Every block contains a barrier.

Proof. Let $B$ be a block: define $B_{1}=\{s \in B \mid \forall t \in B t \not \subset s\}$. By Theorem 1.1(1) there exists a block $B^{\prime}$ such that either $B^{\prime} \subseteq B_{1}$ or $B^{\prime} \cap B_{1}=\varnothing$. In the first case $B^{\prime}$ is a barrier and thus it suffices to show that $B^{\prime} \cap B_{1}=\varnothing$ leads to a contradiction.

If $B^{\prime} \cap B_{1}=\varnothing$ let $s_{0} \in B^{\prime}$ be such that $\operatorname{lh}\left(s_{0}\right)=\min \left\{\operatorname{lh}(s) \mid s \in B^{\prime}\right\}$. Since $s_{0} \notin B_{1}$ there exists $t \in B$ such that $t \subset s$. Obviously $\ln (t)<\ln \left(s_{0}\right)$. Since $B^{\prime} \subseteq B$ is a block we must have $t \in B^{\prime}$, thereby contradicting the minimality of $\operatorname{lh}\left(s_{0}\right)$.

Definition. We define the lexicographic ordering of finite sequences as follows: if $s$ and $t$ are finite sequences $s \leq_{L} t$ if and only if either $s \subseteq t$ or for some $i<\min \{\operatorname{lh}(s), \ln (t)\}$ we have $s[i]=t[i]$ and $s(i)<t(i)$.

Clearly, $\leq_{L}$ is a linear ordering. The following fact was first noticed in [15].

Lemma 1.3. Every block is well-ordered by the lexicographic ordering.

Proof. Let $B$ be a block and suppose $C \subseteq B$ has no minimal element with respect to $\leq_{L}$. Define by recursion a sequence of numbers by

$$
n_{k}=\min \left\{n \mid \exists s \in C\left\langle n_{0}, \ldots, n_{k-1}, n\right\rangle \sqsubset s\right\} .
$$

Since $C$ has no minimal element with respect to $\leq_{L}$ this definition produces an infinite set $X=\left\{n_{k} \mid k \in \mathbb{N}\right\}$. Since $B$ is a block there exists $t \in B$ such that $t \subset X$. This leads to a contradiction with condition (3) in the definition of block.

Definition. If $B$ is a block let o.t. $(B)$ be the ordinal type of the lexicographic ordering of $B$. o.t. $(B)$ is a countable ordinal.

It is obvious that if $B$ and $B^{\prime}$ are blocks such that $B^{\prime} \subseteq B$ then o. t. $\left(B^{\prime}\right) \leq$ o.t. $(B)$. 
While the order type of a block can be any countable limit ordinal the possible order types of barriers are much more restricted, as the following result shows.

Lemma 1.4 [1]. The order types of barriers are exactly the ordinals of the form $\omega^{\beta} \cdot n$ where $0<\beta<\omega_{1}, 0<n<\omega$, and if $\beta<\omega$ then $n=1$.

Since every barrier of order type $\omega^{\beta} \cdot n$ with $n>1$ contains a subbarrier of order type $\omega^{\beta}$, the barriers of indecomposable order type are the most important.

The following definition is a key ingredient in the definition of better quasiordering and was formulated by Nash-Williams in [13].

Definition. Let $s, t \in[\mathbb{N}]^{<\omega}$ : we write $s \triangleleft t$ if there exists $s^{\prime} \in[\mathbb{N}]^{<\omega}$ such that $s \sqsubseteq s^{\prime}$ and $t \sqsubseteq{ }_{*} s^{\prime}$.

Let us remark explicitly that $\triangleleft$ is neither reflexive nor transitive: we have $\langle 3,5,6\rangle \triangleleft\langle 5,6,8,9\rangle \triangleleft\langle 6,8\rangle$ but $\langle 3,5,6\rangle \triangleleft\langle 6,8\rangle$. If $s$ and $t$ are members of a barrier and $s \triangleleft t$ holds then $\ln (s) \leq \operatorname{lh}(t)$.

Definition. If $B$ is a block let $B^{2}=\{s \cup t \mid s, t \in B \wedge s \triangleleft t\} . B^{2}$ is a block and if $t \in B^{2}$ there are unique $\pi_{0}(t), \pi_{1}(t) \in B$ such that $t=\pi_{0}(t) \cup \pi_{1}(t)$ and $\pi_{0}(t) \triangleleft \pi_{1}(t)$.

Notice that if $B$ is a barrier then $B^{2}$ is also a barrier.

Definition. Let $R$ be a binary relation on a set $Q, B$ a block, and $f: B \rightarrow Q$. We say that the map $f$ is good with respect to $R$ if there exist $s, t \in B$ such that $s \triangleleft t$ and $f(s) R f(t)$. If $R$ is clear from the context we suppress its mention. If $f$ is not good then we say that it is bad. $f$ is perfect if for every $s, t \in B$ such that $s \triangleleft t$ we have $f(s) R f(t)$.

Lemma 1.5. Let $R$ be a binary relation on a set $Q, B$ a block (resp. barrier) and $f: B \rightarrow Q$. There exists $B^{\prime} \subseteq B$ such that $B^{\prime}$ is a block (resp. barrier) and $f \mid B^{\prime}$ is either bad or perfect with respect to $R$.

Proof. Let $B_{1}=\left\{t \in B^{2} \mid f\left(\pi_{0}(t)\right) R f\left(\pi_{1}(t)\right)\right\}$ and $B_{2}=B^{2} \backslash B_{1}$. By Theorem 1.1 there exists a block (resp. barrier) $B^{\prime \prime} \subseteq B^{2}$ such that either $B^{\prime \prime} \subseteq B_{1}$ or $B^{\prime \prime} \subseteq B_{2}$. Let $B^{\prime}=\left\{s \in B \mid s \subset\right.$ base $\left.\left(B^{\prime \prime}\right)\right\}: B^{\prime}$ is a block (resp. barrier). If $B^{\prime \prime} \subseteq B_{1}$ then $f \mid B^{\prime}$ is perfect, if $B^{\prime \prime} \subseteq B_{2}$ then $f \mid B^{\prime}$ is bad.

Definition. Let $\alpha$ be a countable ordinal and $R$ a binary relation on a set $Q$. $R$ is an $\alpha$-well relation if for every barrier $B$ with 0. t. $(B) \leq \alpha$, every map $f: B \rightarrow Q$ is good with respect to $R$. If for every $\alpha R$ is an $\alpha$-well relation we say that $R$ is a better relation. If $R$ is a quasi-ordering, i.e., it is reflexive and transitive, the above notions are denoted $\alpha$-wqo and bqo and are the main object of study in this paper. If $\alpha=\omega$ we write just well-relation and wqo.

In light of Lemma 1.2 (and of the fact that every barrier is a block) the definition of $\alpha$-well relation could be given using maps which have as domain a block instead of a barrier (indeed this was Nash-Williams' first definition of bqo, but soon he found out that barriers were more convenient than blocks). In $\S 4$ we will show that we can further restrict ourselves to a particular kind of barriers (the smooth barriers defined in $\S 3$ ) in giving these definitions.

Obviously if $\beta<\alpha$ and $R$ is an $\alpha$-well relation then $R$ is also a $\beta$-well relation. 
Notice that for linear orderings the notions of wqo and bqo coincide with that of well-ordering.

Lemma 1.6. Let $\alpha$ be a countable ordinal and $R$ and $R^{\prime}$ be two $\alpha$-well relations on the same set $Q$. Then the relation $S=R \cap R^{\prime}$ (i.e., the relation such that $q S q^{\prime}$ holds if and only if both $q R q^{\prime}$ and $q R^{\prime} q^{\prime}$ hold) is also an $\alpha$-well relation. Proof. Let $B$ be a barrier with o.t. $(B) \leq \alpha$ and $f: B \rightarrow Q$. By Lemma 1.5 there exists a barrier $B^{\prime} \subseteq B$ such that $f \mid B^{\prime}$ is either bad or perfect with respect to $R$. Since $R$ is an $\alpha$-well relation $f \mid B^{\prime}$ must be perfect with respect to $R$. Since $R^{\prime}$ is an $\alpha$-well relation there exist $s, t \in B^{\prime}$ such that $s \triangleleft t$ and $f(s) R^{\prime} f(t):$ then $f(s) S f(t)$ holds and $f$ is good with respect to $S$.

A basic technique of bqo theory is the so-called minimal bad array lemma, which asserts that if bad maps exist then there exist bad maps with certain minimality properties. This method of proof is originally due to Nash-Williams: for a treatment in which this idea is explicitly isolated see, e.g., [10]. In our study of $\alpha$-wqos we need a version of this result in which only subbarriers of a given barrier are used: this is actually a generalization of one of the earlier results of Nash-Williams [13, Lemma 24].

Definition. Let $(Q, \preceq)$ be a quasi-ordering. A quasi-ordering $\leq$ on $Q$ is compatible with $\preceq$ if it is well-founded and for every $q, q^{\prime} \in Q$ we have $q \leq^{\prime} q^{\prime}$ implies $q \preceq q^{\prime}$. In this situation if $f$ and $g$ are maps from barriers into $Q$ we write $f \leq^{\prime} g$ if $\operatorname{dom}(f) \subseteq \operatorname{dom}(g)$ and $\forall s \in \operatorname{dom}(f)$ $\left(f(s)=g(s) \vee f(s)<^{\prime} g(s)\right)$. We write $f<^{\prime} g$ if $f \leq^{\prime} g$ and $f \neq g \mid \operatorname{dom}(f)$. A map $f$ from a barrier into $Q$ is locally minimal bad with respect to $\leq^{\prime}$ if $f$ is bad (with respect to $\preceq$ ) and there is no $g<^{\prime} f$ which is bad.

The following lemma is our version of the minimal bad array lemma: the proof is an adaptation to our situation of the proof of Theorem 2.19 in [10].

Lemma 1.7. In the situation of the preceding definition if $B$ is a barrier and $f: B \rightarrow Q$ is bad then there exists $g \leq{ }^{\prime} f$ which is locally minimal bad with respect to $\leq$ '.

Proof. We define a sequence of bad maps $f_{n}$ such that $f_{0}=f$ and $f_{n+1}<f_{n}$. If this sequence is finite then its last element is locally minimal bad; otherwise we will define a bad map $f_{\omega}$ such that for all $n f_{\omega}<^{\prime} f_{n}$ and this map will be locally minimal bad. For every $n$ we denote the barrier $\operatorname{dom}\left(f_{n}\right)$ by $B_{n}$.

Suppose $f_{n}$ has been defined: if $f_{n}$ is locally minimal bad the sequence terminates. Otherwise there exists a bad $g_{n}: C_{n} \rightarrow Q$ such that $g_{n}<\prime f_{n}$. We pick $g_{n}$ so that the number $k_{n}=\min \left\{k \mid \exists s \in C_{n}\left(g_{n}(s)<^{\prime} f_{n}(s) \wedge k=s(\operatorname{lh}(s)-1)\right)\right\}$ is as small as possible. Now let $B_{n+1} \subseteq B_{n}$ be the unique barrier such that base $\left(B_{n+1}\right)=\operatorname{base}\left(C_{n}\right) \cup\left\{k \in \operatorname{base}\left(B_{n}\right) \mid k<k_{n}\right\}$. Let $A_{n}=\left\{s \in C_{n}\right.$ $\left.\mid s(\operatorname{lh}(s)-1) \geq k_{n}\right\}$ and define $f_{n+1}: B_{n+1} \rightarrow Q$ as follows:

$$
f_{n+1}(s)= \begin{cases}g_{n}(s) & \text { if } s \in A_{n}, \\ f_{n}(s) & \text { if } s \notin A_{n} .\end{cases}
$$

Clearly $f_{n+1}<^{\prime} f_{n}$. We claim that $f_{n+1}$ is bad. To show this let $s, t \in B_{n+1}$ be such that $s \triangleleft t$. There are three different possibilities.

(1) If $s \in A_{n}$ then also $t \in A_{n}$ and $g_{n}(s) \npreceq g_{n}(t)$ implies $f_{n+1}(s) \npreceq f_{n+1}(t)$. 
(2) If $s \notin A_{n}$ and $t \notin A_{n}$ then $f_{n}(s) \npreceq f_{n}(t)$ implies $f_{n+1}(s) \npreceq f_{n+1}(t)$.

(3) If $s \notin A_{n}$ and $t \in A_{n}$ then $g_{n}(t) \leq^{\prime} f_{n}(t)$ and hence $g_{n}(t) \preceq f_{n}(t)$. On the other hand $f_{n}(s) \npreceq f_{n}(t)$ and hence $f_{n}(s) \npreceq g_{n}(t)$; that is, $f_{n+1}(s) \npreceq f_{n+1}(t)$.

This proves the claim.

Now suppose the sequence $\left\{f_{n}\right\}$ has never terminated. Notice that $k_{n} \leq k_{n+1}$ and each $k \in \mathbb{N}$ can be $k_{n}$ only finitely many times because $\leq^{\prime}$ is well-founded and there are only finitely many $s \in[\mathbb{N}]^{<\omega}$ with $k=s(\operatorname{lh}(s)-1)$. Therefore $\lim _{n \rightarrow \infty} k_{n}=+\infty$.

Let $B_{\omega}=\bigcap_{n} B_{n}$ and define $f_{\omega}: B_{\omega} \rightarrow Q$ by $f_{\omega}(s)=f_{n}(s)$ for any $n$ such that $k_{n}>s(\operatorname{lh}(s)-1)$ and hence $\forall m \geq n f_{m}(s)=f_{n}(s)$.

We claim that $B_{\omega}$ is a barrier. First of all notice that $\forall n k_{n} \in \operatorname{base}\left(B_{\omega}\right)$ and hence base $\left(B_{\omega}\right)=\bigcap_{n}$ base $\left(B_{n}\right)$ is infinite. If $X \subseteq$ base $\left(B_{\omega}\right)$ and $s \in B$ is such that $s \sqsubset X$ then for every $n s \in B_{n}$ and hence $s \in B_{\omega}$. The elements of $B_{\omega}$, which is a subset of $B$, are incomparable under $C$ and this completes the proof of the claim.

To show that $f_{\omega}$ is bad let $s, t \in B_{\omega}$ be such that $s \triangleleft t$. Take $n$ so large that $f_{n}(s)=f_{\omega}(s)$ and $f_{n}(t)=f_{\omega}(t)$. Since $f_{n}$ is bad we have $f_{\omega}(s) \npreceq f_{\omega}(t)$.

Now suppose $g$ is bad and $g<^{\prime} f_{\omega}$ : for some $s \in \operatorname{dom}(g)$ we have $g(s)<^{\prime}$ $f_{\omega}(s)$. Let $k=s(\operatorname{lh}(s)-1)$ : for some $n$ we have $k_{n}>k$ and (since $\left.g<^{\prime} f_{n}\right)$ the choice of $g_{n}$ did not respect the minimality of $k_{n}$. This contradiction proves that $f_{\omega}$ is locally minimal bad and completes the proof of the lemma.

The following result is due to Pouzet [17, Theorem 7] and allows us to shift from a binary relation which is $\alpha$-well to a quasi-ordering which is $\alpha$-wqo. Therefore whenever we need to find a quasi-ordering having certain properties with respect to the notion of $\alpha$-wqo it suffices to find a (not necessarily transitive) binary relation having the same properties with respect to the notion of $\alpha$-well relation. The possibility of studying arbitrary binary relations in place of quasi-orderings in bqo theory was mentioned by Shelah in the introduction of [20], but to our knowledge has never been developed.

Theorem 1.8. Let $R$ be a reflexive binary relation on a countable set $Q$. There exists a partial ordering $\preceq$ of the set $Q$ which is uniformly primitive recursive in $Q$ and $R$ and satisfies

(1) $\preceq \subseteq R$ (as subsets of $Q \times Q$ );

(2) for every countable ordinal $\alpha, \preceq$ is $\alpha$-wqo if and only if $R$ is an $\alpha$-well relation.

Proof. Let $\left\{q_{n}\right\}$ be an enumeration of $Q$ and let $\#: Q \rightarrow \mathbb{N}$ be the function such that $\#\left(q_{n}\right)=n$. We define $\preceq$ by primitive recursion as follows: suppose $m \leq n$ and we have already established whether $q_{i} \preceq q_{j}$ for all $i<m$ and $j \leq$ $n$ and set $q_{m} \preceq q_{n}$ if and only if $q_{m} R q_{n}$ and $\forall i<m\left(q_{i} \preceq q_{m} \longrightarrow q_{i} \preceq q_{n}\right)$. If $m>n$ then $q_{m} \preceq q_{n}$ never holds.

It is clear that $\preceq$ is a partial ordering uniformly primitive recursive in $Q$ and $R$ and that $\preceq \subseteq R$. For any countable ordinal $\alpha$ if $\preceq$ is $\alpha$-wqo then (since $\preceq \subseteq R$ ) $R$ is an $\alpha$-well relation.

Now suppose $\preceq$ is not $\alpha$-wqo and let $B$ be a barrier with o.t. $(B) \leq \alpha$ and $f: B \rightarrow Q$ be a bad map with respect to $\preceq$. $\preceq$ is well-founded and hence it is a partial ordering compatible with itself. By Lemma 1.7 there exists a barrier 
$B^{\prime} \subseteq B$ and a locally minimal bad map $f^{\prime}: B^{\prime} \rightarrow Q$ such that $\forall s \in B^{\prime} f^{\prime}(s) \preceq$ $f(s)$. Define a map $h: B^{\prime} \rightarrow[Q]^{<\omega}$ by $h(s)=\left\{q \mid q \prec f^{\prime}(s)\right\} . h(s) \in[Q]^{<\omega}$ because it has at most \#(f'(s)) elements. On $[Q]^{<\omega}$ we define a quasi-ordering $\Omega^{*}$ by setting $X \preceq^{*} Y$ if and only if there exists a one-to-one map $G: X \rightarrow Y$ such that for all $q \in X q \preceq G(q)$.

Sublemma. There exists a barrier $B^{\prime \prime} \subseteq B^{\prime}$ such that $h \backslash B^{\prime \prime}$ is perfect with respect to $\preceq^{*}$.

Proof. By Lemma 1.5 there exists a barrier $B^{\prime \prime} \subseteq B^{\prime}$ on which $h$ is either bad or perfect. Suppose $h\left\lceil B^{\prime \prime}\right.$ is bad and consider the partial ordering given by $\subseteq$ on $[Q]^{<\omega}$ : it is clearly compatible with $\Omega^{*}$. Hence there is $h^{\prime}$ which is below $h$ and locally minimal bad with respect to $\subseteq$. Let $C=\operatorname{dom}\left(h^{\prime}\right)$. For any $s \in C$ let $g(s)$ be maximal (with respect to $\preceq$ ) in $h^{\prime}(s)$ and set $h^{\prime \prime}(s)=h^{\prime}(s) \backslash\{g(s)\}$. Since $h^{\prime}$ is locally minimal bad $h^{\prime \prime}$ is perfect on some barrier $C^{\prime} \subseteq C$ and therefore $g: C^{\prime} \rightarrow Q$ is bad with respect to $\preceq$. Moreover for all $s \in C^{\prime}$ we have $g(s) \in h^{\prime}(s) \subseteq h(s)$ and hence $g(s) \prec f^{\prime}(s)$ : this contradicts the minimality of $f^{\prime}$ and therefore $h \backslash B^{\prime \prime}$ is perfect.

Take $B^{\prime \prime}$ satisfying the sublemma and consider $f^{\prime}: B^{\prime \prime} \rightarrow Q$. Suppose towards a contradiction that $R$ is an $\alpha$-well relation: then by Lemma 1.6 the relation $R \cap\left\{\left(q, q^{\prime}\right) \mid \#(q) \leq \#\left(q^{\prime}\right)\right\}$ is also an $\alpha$-well relation on $Q$. Therefore there exist $s, t \in B^{\prime \prime}$ such that $s \triangleleft t, f^{\prime}(s) R f^{\prime}(t)$ and $\#\left(f^{\prime}(s)\right) \leq \#\left(f^{\prime}(t)\right)$. Since $h(s) \preceq^{*} h(t)$ we then have that $\forall i<\#\left(f^{\prime}(s)\right)\left(q_{i} \preceq f^{\prime}(s) \rightarrow q_{i} \preceq f^{\prime}(t)\right)$. Hence the second condition in the definition of $\preceq$ is satisfied and $f^{\prime}(s) \preceq f^{\prime}(t)$ holds. Therefore $f^{\prime}$ is good, a contradiction.

Corollary 1.9. Let $R$ be a reflexive binary relation on a countable set $Q$. There exists a partial ordering $\preceq \subseteq R$ of the set $Q$ which is uniformly primitive recursive in $Q$ and $R$ and is $a$ bqo if and only if $R$ is a better relation.

Proof. Immediate from the theorem.

Notice that the only use of the fact that our enumeration of $Q$ is in order type $\omega$ in the proof of Theorem 1.8 is to guarantee that $h(s) \in[Q]^{<\omega}$. If we can enumerate $Q$ as $\left\{q_{\alpha}\right\}_{\alpha<\beta}$ for some (possibly uncountable) ordinal $\beta$ in a way such that for every $\alpha<\beta$ the set $\left\{\alpha^{\prime}<\alpha \mid q_{\alpha^{\prime}} R q_{\alpha}\right\}$ is finite, the proof works as well and we get a partial ordering $\preceq$ compatible with $\left\{q_{\alpha}\right\}$ in the sense that $q_{\alpha} \preceq q_{\alpha^{\prime}}$ implies $\alpha \leq \alpha^{\prime}$ (obviously in this case $\preceq$ is primitive recursive also in this enumeration).

Pouzet [17] has constructed binary relations on uncountable sets for which Theorem 1.8 fails but has shown that corollary 1.9 holds also in the uncountable case.

\section{2. $Q \alpha$-WQO IMPLIES $Q^{<\alpha}$ WQO}

Definition. If $\left(Q, \leq_{Q}\right)$ is a quasi-ordering let $\tilde{Q}$ be the set of all transfinite countable sequences of elements of $Q$. We extend to the elements of $\tilde{Q}$ the notations we used for finite sequences: for example if $\sigma \in \tilde{Q}$ we have that $\ln (\sigma)$ is a countable ordinal. If $\alpha$ is a countable ordinal let $Q^{<\alpha}=\{\sigma \in \tilde{Q} \mid$ $\operatorname{lh}(\sigma)<\alpha\} . \tilde{Q}$ is quasi-ordered by the embeddability relation: $\sigma \leq \sigma^{\prime}$ if and only if there exists a strictly increasing map $f: \ln (\sigma) \rightarrow \ln \left(\sigma^{\prime}\right)$ such that for every $\beta<\ln (\sigma)$ we have $\sigma(\beta) \leq_{Q} \sigma^{\prime}(f(\beta))$. 
One of the very first results of bqo theory is Nash-Williams' theorem asserting that if $Q$ is bqo then $\tilde{Q}$ is wqo [14]. The main goal of this section is to prove that this theorem holds stage by stage, namely that for any countable indecomposable ordinal $\alpha$, if $Q$ is $\alpha$-wqo then $Q^{<\alpha}$ is wqo (the reverse implication is due to Pouzet [15]). We will prove this result by establishing a chain of implications starting with the following lemma, which for $\alpha=\omega$ is a basic result of wqo theory known as Higman's theorem. Throughout this section $\alpha$ will be a fixed countable indecomposable ordinal.

Lemma 2.1. If $Q$ is $\alpha$-wqo then $Q^{<\omega}$ is $\alpha$-wqo.

Proof. Suppose $Q^{<\omega}$ is not $\alpha$-wqo and let $f: B \rightarrow Q^{<\omega}$ be a bad mapping with o.t. $(B) \leq \alpha$. On $Q^{<\omega} \sqsubseteq$ is well-founded and compatible with $\leq$. Hence we may assume, by Lemma 1.7 , that $f$ is locally minimal bad with respect to 드.

Clearly $\forall s \in B \operatorname{lh}(f(s))>0$ and we can define maps $f^{\prime}: B \rightarrow Q^{<\omega}$ and $g: B \rightarrow Q$ such that for every $s \in B$ we have $\left.\left.f(s)=f^{\prime}(s)\right\} g(s)\right\rangle$. Since $Q$ is $\alpha$-wqo Lemma 1.5 implies that there exists a barrier $B^{\prime} \subseteq B$ on which $g$ is perfect. The badness of $f$ then requires $f^{\prime} \mid B^{\prime}$ to be bad, contradicting the minimality of $f$.

We now introduce two subsets of $\tilde{Q}$ which are extremely useful for our proof. Definition. If $\sigma \in \tilde{Q}$ a tail of $\sigma$ is a sequence of the form $\langle\sigma(\gamma)\rangle_{\beta \leq \gamma<\operatorname{lh}(\sigma)}$ for some $\beta<\operatorname{lh}(\sigma)$. A sequence $\sigma \in \tilde{Q}$ is said to be indecomposable if it can be embedded in each of its tails. The set of all indecomposable sequences is denoted by $\mathscr{I}$.

It is immediate to see that if $\sigma \in \mathscr{I}$ then $\operatorname{lh}(\sigma)$ is an indecomposable ordinal.

Definition. We define a set $\mathscr{H} \subseteq \tilde{Q}$ by the following inductive definition: the sequence of length 0 and all sequences of length 1 are in $\mathscr{H}$ and if $\left\langle\sigma_{n}\right\rangle_{n<\omega}$ is a sequence of elements of $\mathscr{Z}$ such that $\forall n \exists m>n \sigma_{n} \leq \sigma_{m}$ (such a sequence is called quasi-monotonic) then the sequence $\sum_{n} \sigma_{n}=\sigma_{0} \wedge \sigma_{1} \ldots{ }^{\wedge} \sigma_{n} \wedge \ldots$ is also in $\mathscr{H}$. The elements of $\mathscr{H}$ are called hereditarily indecomposable sequences.

The hereditarily indecomposable sequences are analogous to the hereditarily additively indecomposable countable linear orderings introduced by Laver in [6] and used by Rosenstein $[19, \S 10.5]$ in his exposition of the proof of Fraissé's conjecture. Indeed the proofs of Lemmas 2.5 and 2.7 (but not that of Lemma 2.4) are just translations of the corresponding proofs given by Rosenstein for linear orderings.

An easy induction on the definition of $\mathscr{H}$ shows that $\mathscr{H} \subseteq \mathscr{I}$. A corollary of our results will be that if $Q$ is bqo then $\mathscr{H}=\mathscr{I}$ holds.

Definition. If $\alpha$ is a countable ordinal we denote by $\mathscr{H}_{<\alpha}$ the set $\mathscr{H} \cap Q^{<\alpha}$.

Lemma 2.2. Suppose $\sigma, \tau \in \tilde{Q}$ are such that $\sigma=\sum_{n} \sigma_{n}, \tau=\sum_{n} \tau_{n}$, the second sum is quasi-monotonic and $\sigma \leq \tau$. Then there exists $n_{0}$ such that for all $m$ we have $\sigma_{n_{0}} \leq \sum_{n \leq m} \tau_{n}$.

Proof. Suppose that for every $n_{0}$ there is $m$ such that $\sigma_{n_{0}} \leq \sum_{n<m} \tau_{n}$ : using the quasi-monotonocity of the sequence $\left\langle\tau_{n}\right\rangle_{n<\omega}$ we have that for every $n_{0}$ 
and $i$ there exists $m$ such that $\sigma_{n_{0}} \leq \sum_{i<n \leq m} \tau_{n}$. This implies that $\sigma \leq \tau$, a contradiction.

To perform the next step in our chain of implications we will also need a simple combinatorial fact relating the width of an $\omega$-branching well-founded tree, as expressed by the lexicographic order type of its endnodes, to its height.

Definition. If $T \subseteq[N]^{<\omega}$ is a tree we denote by $L_{T}$ the set of its leaves or endnodes, i.e., $\left.\left.L_{T}=\{s \in T \mid \forall n s\} n\right\rangle \notin T\right\}$. If $T$ is well-founded let $\operatorname{ht}(T)$ be the countable ordinal representing the height of $T$, namely $h t(T)=\mathrm{ht}_{T}(\langle\rangle)$ where $\mathrm{ht}_{T}$ is defined recursively on $T$ by

$$
\operatorname{ht}_{T}(s)=\sup \left\{\operatorname{ht}_{T}\left(s^{\mathcal{\imath}}\langle n\rangle\right)+1 \mid s^{\mathcal{\gamma}}\langle n\rangle \in T\right\} .
$$

Lemma 2.3. If $T$ is a well-founded tree and $\operatorname{ht}(T) \leq \beta$ then o.t. $\left(L_{T}\right) \leq \omega^{\beta}$. Proof. Straightforward induction on $\beta$.

The core of our proof is contained in the following lemma.

Lemma 2.4. If $Q^{<\omega}$ is $\alpha$-wqo then $\mathscr{H}_{<\alpha}$ is wqo.

Proof. Suppose $\mathscr{K}_{<\alpha}$ is not wqo and let $f: \mathbb{N} \rightarrow \mathscr{K}_{<\alpha}$ be a bad sequence. We will define a sequence of bad maps $f_{k}: B_{k} \rightarrow \mathscr{K}_{<\alpha} \cup Q^{<\omega}$, where $B_{k}$ is a block with base $\left(B_{k}\right)=\mathbf{N}$. We start with $B_{0}=[\mathbb{N}]^{1}$ and $f_{0}=f$ (we identify an element of $\mathbf{N}$ with the singleton containing it).

Suppose that we have defined $f_{k}$ and $B_{k}$ and let

$$
C_{k}=\left\{s \in B_{k} \mid f_{k}(s) \in Q^{<\omega}\right\}, \quad D_{k}=\left\{s \in B_{k}^{2} \mid f_{k}\left(\pi_{0}(s)\right) \notin Q^{<\omega}\right\} .
$$

Set $B_{k+1}=C_{k} \cup D_{k}$. It is immediate to check that that $B_{k+1}$ is a block and base $\left(B_{k+1}\right)=N$. We define $f_{k+1}: B_{k+1} \rightarrow \mathscr{K}_{<\alpha} \cup Q^{<\omega}$ by cases as follows:

(1) If $s \in C_{k}$ let $f_{k+1}(s)=f_{k}(s)$.

(2) If $s \in D_{k}$ and $f_{k}\left(\pi_{1}(s)\right) \notin Q^{<\omega}$ we can write $f_{k}\left(\pi_{0}(s)\right)=\sum_{n} \sigma_{n}$ and $f_{k}\left(\pi_{1}(s)\right)=\sum_{n} \tau_{n}$ with both sums quasi-monotonic. Since $f_{k}$ is bad and $\pi_{0}(s) \triangleleft \pi_{1}(s)$ we have $\sum_{n} \sigma_{n} \not \sum \sum_{n} \tau_{n}$ and by Lemma 2.2 there exists $n_{0}$ such that for every $m$ we have $\sigma_{n_{0}} \not \sum_{n \leq m} \tau_{n}$. Let $f_{k+1}(s)=\sigma_{n_{0}}$.

(3) If $s \in D_{k}$ and $f_{k}\left(\pi_{1}(s)\right) \in Q^{<\omega}$ let $n=\ln \left(f_{k}\left(\pi_{1}(s)\right)\right)$. Let $f_{k+1}(s)=$ $f_{k}\left(\pi_{0}(s)\right)[n+1]$ (so that $\left.\ln \left(f_{k+1}(s)\right)=n+1\right)$.

We claim that with this definition $f_{k+1}$ is bad. To see this suppose $s, t \in$ $B_{k+1}$ and $s \triangleleft t$. We need to distinguish four different cases:

(1) If $s, t \in C_{k}$ then $f_{k+1}(s)=f_{k}(s) \not f_{k}(t)=f_{k+1}(t)$.

(2) If $s \in C_{k}$ and $t \in D_{k}$ then $s \triangleleft \pi_{0}(t)$ and hence $f_{k}(s) \not f_{k}\left(\pi_{0}(t)\right)$. Moreover $f_{k+1}(t) \leq f_{k}\left(\pi_{0}(t)\right)$ and therefore $f_{k+1}(s)=f_{k}(s) \notin f_{k+1}(t)$.

(3) If $s \in D_{k}$ and $t \in C_{k}$ we have $\pi_{1}(s)=t$ and hence in defining $f_{k+1}(s)$ we use condition (3). Therefore $\operatorname{lh}\left(f_{k+1}(s)\right)>\operatorname{lh}\left(f_{k}(t)\right)=\operatorname{lh}\left(f_{k+1}(t)\right)$ and we necessarily have $f_{k+1}(s)\left\lfloor f_{k+1}(t)\right.$.

(4) If $s, t \in D_{k}$ we have $\pi_{1}(s)=\pi_{0}(t)$ and hence $f_{k+1}(s)$ is defined using condition (2). Therefore if $f_{k}\left(\pi_{0}(t)\right)=\sum_{n} \tau_{n}$ for every $m$ we have that $f_{k+1}(s) \notin \sum_{n \leq m} \tau_{n}$. On the other hand it is clear that for some $m$ $f_{k+1}(t) \leq \sum_{n \leq m} \tau_{n}$ : these two facts imply $f_{k+1}(s)\left\lfloor f_{k+1}(t)\right.$. 
This completes the proof of the claim and our construction of the sequence of bad maps.

Notice that if $X \in[\mathbb{N}]^{\omega}$ and for every $k$ we define $s_{k}(X)$ to be the unique sequence such that $s_{k}(X) \in B_{k}$ and $s_{k}(X) \sqsubset X$, we have either $s_{k}(X) \in C_{k}$ or $\ln \left(f_{k+1}\left(s_{k+1}(X)\right)\right)<\ln \left(f_{k}\left(s_{k}(X)\right)\right)$.

Now let us define $B=\bigcup_{k} C_{k}$. We claim that $B$ is a block. To see this notice that, by the above observation, for any $X \in[\mathbb{N}]^{\omega}$ there exists $k$ such that $s_{k}(X) \in C_{k}$ : this implies that base $(B)=\mathbb{N}$ and that condition (2) in the definition of block is satisfied. Condition (3) follows from the fact that if $s_{k}(X) \in C_{k}$ then for all $k^{\prime}>k$ we have $s_{k^{\prime}}(X)=s_{k}(X)$.

Define $f_{\omega}: B \rightarrow Q^{<\omega}$ by setting $f_{\omega}(s)=f_{k}(s)$ for the least (or any) $k$ such that $s \in C_{k}$. It is immediate to check that $f_{\omega}$ is bad and hence if $B^{\prime} \subseteq B$ is the barrier given by Lemma $1.2 f_{\omega} \mid B^{\prime}$ is also bad. Therefore to complete the proof of the lemma it suffices to show that o.t. $(B)<\alpha$, because then 0.t. $\left(B^{\prime}\right)<\alpha$ as well. We begin by proving a couple of simple facts about our construction.

Sublemma 1. If $s \in B_{k}$ then $\operatorname{lh}(s) \leq k+1$.

Proof. By induction on $k$. If $k=0$ this is obvious. If $s \in B_{k+1}$ either $s \in$ $C_{k} \subseteq B_{k}$ and hence $\operatorname{lh}(s) \leq k+1<k+2$ by induction hypothesis, or $s \in D_{k} \subseteq$ $B_{k}^{2}$ and $\pi_{0}(s), \pi_{1}(s) \in B_{k}$ : hence $\left.\operatorname{lh}(s)=\max \left(\operatorname{lh}\left(\pi_{0}(s)\right), \operatorname{lh}\left(\pi_{1}(s)\right)+1\right)\right) \leq$ $k+2$.

Sublemma 2. If $s \in B_{k}$ and $\ln (s)<k+1$ then $s \in C_{k}$ and hence $\forall k^{\prime}>k$ $s \in B_{k^{\prime}}$.

Proof. By induction on $k$. If $k=0$ the hypothesis never holds. Suppose $s \in B_{k+1}$ and $\operatorname{lh}(s)<k+2$. If $s \in C_{k}$ then also $s \in C_{k+1}$. If $s \in D_{k}$ we have $\pi_{0}(s) \notin C_{k}$ and by the induction hypothesis and Sublemma $1 \operatorname{lh}\left(\pi_{0}(s)\right)=k+1$. Since $\operatorname{lh}(s) \leq \operatorname{lh}\left(\pi_{0}(s)\right)$ we have $s=\pi_{0}(s)$ and therefore $\pi_{1}(s) \subset s$. Therefore $\operatorname{lh}\left(\pi_{1}(s)\right)<k+1$ and, by the induction hypothesis, $\pi_{1}(s) \in C_{k}$. But then in defining $f_{k+1}(s)$ we use condition (3) and $f_{k+1}(s) \in Q^{<\omega}$. Therefore $s \in C_{k+1}$ also in this case and the proof is complete.

Consider the set $T=\{(\rangle\} \cup \bigcup_{k} B_{k}$ : we claim that $T$ is a tree. Let $s \in T$ and suppose $s \in B_{k}$ : by Sublemma $1 \operatorname{lh}(s) \leq k+1$. We prove by induction on $i<\operatorname{lh}(s)$ that $s[i+1] \in B_{i} \subset T$. For $i=0$ it is clear that $s[1] \in B_{0}$. If $i<\operatorname{lh}(s)-1$ and $s[i+1] \in B_{i}$ but $s[i+2] \notin B_{i+1}$ then, since $B_{i+1}$ is a block, Sublemma 1 implies that $s[i+1] \in B_{i+1}$. Sublemma 2 then implies that $s[i+1] \in C_{i+1}$ and hence $s[i+1] \in B_{k}$ against $s \in B_{k}$ (since $\left.s[i+1] \sqsubset s\right)$. This completes the proof of our claim.

$T$ is well-founded by the same argument which showed that $B$ is a block and indeed $L_{T}=B$. To prove that o.t. $(B)<\alpha$ we will use Lemma 2.3 and thus it is convenient to write $\alpha=\omega^{\beta}$. Then it suffices to show that $h t(T) \leq \beta$. To this end define an ordinal valued function $g$ on $T$ by

$$
g(s)= \begin{cases}\beta & \text { if } s=\langle\rangle, \\ \gamma & \text { if for some } k s \in B_{k} \backslash B \text { and } \operatorname{lh}\left(f_{k}(s)\right)=\omega^{\gamma}, \\ 0 & \text { if } s \in B .\end{cases}
$$

Then by induction on $T$ we can prove $\forall s \in T \mathrm{ht}_{T}(s) \leq g(s)$ and hence ht $(T) \leq \beta$. 
The last step in our chain of implications is provided by the following lemmas, which emphasize the importance of hereditarily indecomposable sequences.

Lemma 2.5. If $\mathscr{K}_{<\alpha}$ is wqo then every element of $Q^{<\alpha}$ can be written as the concatenation of finitely many elements of $\mathscr{H}$.

Proof. By Cantor's normal form theorem for ordinals it suffices to prove the lemma for $\sigma$ such that $\ln (\sigma)=\omega^{\beta}<\alpha$. We prove this by induction on $\beta$. The lemma is obvious when $\beta=0$. Given $\sigma$ with $\operatorname{lh}(\sigma)=\omega^{\beta}>1$ we can write $\sigma=\sum_{n} \sigma_{n}$ where for each $n$ we have $\operatorname{lh}\left(\sigma_{n}\right)<\omega^{\beta}$. By the induction hypothesis every $\sigma_{n}$ can be written as the concatenation of finitely many elements of $\mathscr{H}$ and hence we can assume that for every $n$ we have $\sigma_{n} \in \mathscr{H}$.

We claim that there exists $m$ such that $\forall n>m \exists n^{\prime}>n \sigma_{n} \leq \sigma_{n^{\prime}}$. Indeed if this is not the case we have $\forall m \exists n>m \forall n^{\prime}>n \sigma_{n} \not \sigma_{n^{\prime}}$ and it is easy to extract from $\left\langle\sigma_{n}\right\rangle$ a bad subsequence, against the fact that $\mathscr{K}_{<\alpha}$ is wqo.

The claim we just proved shows that for some $m$ the sequence $\left\langle\sigma_{n}\right\rangle_{n>m}$ is quasi-monotonic and hence $\sum_{n>m} \sigma_{n} \in \mathscr{H}$. Therefore $\sigma_{0} \wedge^{\cdots} \wedge^{\wedge} \sigma_{m} \wedge\left(\sum_{n>m} \sigma_{n}\right)$ expresses $\sigma$ as the concatenation of finitely many elements of $\mathscr{H}$.

Corollary 2.6. If $Q$ is $\alpha$-wqo then $\mathscr{X}_{<\alpha}=Q^{<\alpha} \cap \mathscr{I}$. If $Q$ is bqo then $\mathscr{H}=\mathscr{I}$.

Proof. The second statement clearly follows from the first.

It is clear that $\mathscr{H}_{<\alpha} \subseteq Q^{<\alpha} \cap \mathscr{I}$. Let $\sigma \in Q^{<\alpha} \cap \mathscr{I}$ : we need to prove that $\sigma \in \mathscr{H}$. By Lemmas 2.1, 2.4, and 2.5 we can write $\left.\sigma=\sigma_{0}\right\urcorner \ldots \wedge \sigma_{n}$ with $\sigma_{i} \in \mathscr{H}$. Suppose $n>0$ (otherwise $\sigma \in \mathscr{H}$ immediately): since $\sigma \in$ $\mathcal{I}$ we have $\operatorname{lh}\left(\sigma_{n}\right)>1$ and hence we can write $\sigma_{n}=\sum_{m} \tau_{m}$ as a quasimonotonic sum of elements of $\mathscr{H}$. Since $\sigma$ is indecomposable $\sigma \leq \sigma_{n}$ and hence for each $i<n$ there exists $m_{i}$ such that $\sigma_{i} \leq \sum_{m<m_{i}} \tau_{m}$. Let $f_{i}$ be an embedding witnessing this and let $m<m_{i}$ be the largest $m$ such that $\operatorname{rng}\left(f_{i}\right) \cap \operatorname{dom}\left(\tau_{m}\right) \neq \varnothing$. Then a tail of $\sigma_{i}$ embeds in $\tau_{m}$ and since $\sigma_{i} \in \mathscr{H} \subseteq \mathscr{J}$ we have $\sigma_{i} \leq \tau_{m}$. Therefore the sequence $\left\langle\sigma_{0}, \ldots, \sigma_{n-1}, \tau_{0}, \tau_{1}, \ldots\right\rangle$ is quasimonotonic and $\sigma=\sigma_{0} \wedge^{\wedge} \wedge_{n-1} \wedge\left(\sum_{m} \tau_{m}\right) \in \mathscr{H}$.

Lemma 2.7. If $\mathscr{H}_{<\alpha}$ is wqo then $Q^{<\alpha}$ is wqo.

Proof. If $\left\langle\sigma_{n}\right\rangle$ is a sequence in $Q^{<\alpha}$ for every $n$ we can write, by Lemma 2.5, $\sigma_{n}=\tau_{n, 0} \wedge \ldots \wedge \tau_{n, k_{n}}$ where $\tau_{n, i} \in \mathscr{K}_{<\alpha}$. Since $\mathscr{K}_{<\alpha}$ is wqo Lemma 2.1 implies that $\left(\mathscr{H}_{<\alpha}\right)^{<\omega}$ is also wqo: hence there exist $n<m$ such that $\left\langle\tau_{n, 0}, \ldots, \tau_{n, k_{n}}\right\rangle$ $\leq\left\langle\tau_{m, 0}, \ldots, \tau_{m, k_{m}}\right\rangle$ (this is just embeddability between finite sequences) and therefore $\sigma_{n} \leq \sigma_{m}$.

The following is the main result of this section.

Theorem 2.8. For any indecomposable ordinal $\alpha$ and any quasi-ordering $Q, Q$ is $\alpha$-wqo if and only if $Q^{<\alpha}$ is wqo.

Proof. If $Q$ is $\alpha$-wqo Lemmas 2.1, 2.4 and 2.7 show that $Q^{<\alpha}$ is wqo. For the reverse implication see [15, III-4.1] or [4, §8.5].

Corollary 2.9 [15]. A quasi-ordering $Q$ is bqo if and only if $\tilde{Q}$ is wqo.

Proof. Immediate from the theorem, since $\tilde{Q}$ is wqo if and only if for every countable $\alpha Q^{<\alpha}$ is wqo. 
The techniques employed in the proof of Theorem 2.8, and in particular in the proof of Lemma 2.4, can be generalized to yield a result of the following form, whose proof we leave to the reader:

Theorem 2.10. For some ordinal valued function on pairs of countable ordinals $\mu$ we have: if $Q$ is $\omega^{\mu(\alpha, \beta)}$-wqo then $Q^{<\omega^{\alpha}}$ is $\omega^{\beta}$-wqo. A rough estimate on $\mu$ is given by $\mu(\alpha, \beta) \leq \beta \cdot(\alpha+1)$.

Corollary 2.11 [14]. If $Q$ is bqo then $\tilde{Q}$ is bqo.

Proof. Immediate from the theorem.

\section{3. $\alpha$-WQOS WHICH ARE NOT BQOS}

This section is devoted to the construction of partial orderings which show that the notions of $\alpha$-wqo for different indecomposable $\alpha$ are all distinct, i.e., that if $\beta<\alpha$ are countable indecomposable ordinals there exists a partial ordering which is $\beta$-wqo but not $\alpha$-wqo (the quasi-orderings defined to this end in [15] are incorrect for $\alpha>\omega^{\omega}$ ). This shows also that for no countable $\alpha$ the notion of $\alpha$-wqo is equivalent to that of bqo. Combining this result with Theorem 2.8 we obtain also that for every countable indecomposable $\alpha$ there exists a partial ordering $Q$ such that $Q^{<\alpha}$ is wqo but $\tilde{Q}$ is not wqo.

If $Q$ is a quasi-ordering which is not $\alpha$-wqo but is $\beta$-wqo for every $\beta<\alpha$ we can use the bad map showing that $Q$ is not $\alpha$-wqo to define a quasi-ordering on a barrier $B$ of order type $\alpha$ which lies in the complement of $\triangleleft$ (in $B \times B$ ) and is $\beta$-wqo for every $\beta<\alpha$. In light of Theorem 1.8 it suffices to find a binary relation with these properties and hence it is enough to show that the complement of $\triangleleft$ on a barrier $B$ of order type $\alpha$ is a $\beta$-well relation for every $\beta<\alpha$. It is clear that some barriers do not have this property (if $B$ has a subbarrier of smaller order type the property fails), but we will show that barriers satisfying an additional condition have this property.

For notational convenience throughout this section we assume that all the blocks $B$ we consider are such that base $(B)=\mathbb{N}$. This is not restrictive since every block $B$ is isomorphic to a block with base $\mathbb{N}$ via the increasing enumeration of base $(B)$.

Definition. A set $C \subseteq[\mathbb{N}]^{<\omega}$ is smooth if for all $s, s^{\prime} \in C$ such that $\operatorname{lh}(s)<$ $\operatorname{lh}\left(s^{\prime}\right)$ there exists $i<\operatorname{lh}(s)$ such that $s(i)<s^{\prime}(i)$.

Notice that the elements of a smooth set are incomparable under $\subset$. Therefore every smooth block is a barrier and if $C$ is a smooth set and $s, s^{\prime} \in C$ are such that $s \triangleleft s^{\prime}$ then $\ln (s) \leq \operatorname{lh}\left(s^{\prime}\right)$.

The following alternative characterization of smoothness may give a more intuitive feeling for this concept: suppose the elements of $C$ are incomparable under $ᄃ$ and define a partial function $\Phi_{C}:[\mathbb{N}]^{\omega} \rightarrow \mathbb{N}$ by letting $\Phi_{C}(X)=n$ if and only if $X[n] \in C$. Then $C$ is smooth if and only if

$$
\begin{aligned}
\forall X, X^{\prime} \in[\mathbb{N}]^{\omega}\left(\Phi_{C}(X), \Phi_{C}\left(X^{\prime}\right) \text { exist } \wedge \forall i X(i) \leq X^{\prime}(i)\right. \\
\left.\longrightarrow \Phi_{C}(X) \leq \Phi_{C}\left(X^{\prime}\right)\right) .
\end{aligned}
$$

Lemma 3.1. For any countable indecomposable ordinal $\alpha$ there exists a smooth barrier $B$ such that o.t. $(B)=\alpha$. 
Proof. An easy induction shows that all the barriers defined in part III.2.6 of [15] are smooth.

Lemma 3.2. If $B$ and $B^{\prime}$ are blocks such that o.t. $(B)<0 . t .\left(B^{\prime}\right)$ there exist $s \in B$ and $s^{\prime} \in B^{\prime}$ such that $s \sqsubset s^{\prime}$.

Proof. If the conclusion fails, for any $s^{\prime} \in B^{\prime}$ there exists $g\left(s^{\prime}\right) \in B$ such that $s^{\prime} \sqsubseteq g\left(s^{\prime}\right)$. The map $g: B^{\prime} \rightarrow B$ clearly preserves the lexicographic order and shows o.t. $\left(B^{\prime}\right) \leq$ o.t. $(B)$.

A map which is bad with respect to the negation of $\triangleleft$ is a map $f$ which preserves $\triangleleft$, i.e., such that $s \triangleleft t$ implies $f(s) \triangleleft f(t)$. The following lemma about these maps will be useful also in $\$ 5$.

Lemma 3.3. Let $B$ be a barrier and $C$ a smooth subset of $[\mathbb{N}]^{<\omega}$. If $t \in B$ and $f: B \rightarrow C$ preserves $\triangleleft$ then we have

(1) $\operatorname{lh}(f(t)) \leq \operatorname{lh}(t)$

(2) $\forall i<\ln (f(t)) t(i) \leq f(t)(i)$.

Proof. (1) Let $\operatorname{lh}(t)=k$ and for every $i<k$ let $t_{i}, t_{i}^{\prime} \in B$ be such that

$$
\begin{aligned}
& t_{i} \sqsubset\langle t(i), \ldots, t(k-1), t(k-1)+1, t(k-1)+2, \ldots\rangle, \\
& t_{i}^{\prime} \sqsubset\langle t(i), \ldots, t(k-1), t(k-1)+2, t(k-1)+3, \ldots\rangle .
\end{aligned}
$$

Moreover let $t_{k}, t_{k}^{\prime} \in B$ be such that

$t_{k} \sqsubset\langle t(k-1)+1, t(k-1)+2, \ldots\rangle, \quad t_{k}^{\prime} \sqsubset\langle t(k-1)+2, t(k-1)+3, \ldots\rangle$.

Therefore $t=t_{0}=t_{0}^{\prime}, t_{k} \triangleleft t_{k}^{\prime}$, and for every $i<k \quad t_{i} \triangleleft t_{i+1}$ and $t_{i}^{\prime} \triangleleft t_{i+1}^{\prime}$. Suppose that $\operatorname{lh}(f(t))>k$ so that $f(t)(k)$ exists: since $f$ preserves $\triangleleft$ and $C$ is smooth (insuring that all the sequences involved are long enough) we have

$$
\begin{gathered}
f(t)(k)=f\left(t_{1}\right)(k-1)=\cdots=f\left(t_{k}\right)(0), \\
f(t)(k)=f\left(t_{1}^{\prime}\right)(k-1)=\cdots=f\left(t_{k}^{\prime}\right)(0)
\end{gathered}
$$

against $f\left(t_{k}\right) \triangleleft f\left(t_{k}^{\prime}\right)$.

(2) We prove this simultaneously for all $t \in B$ by induction on $i$. If $i=0$ for every $j \leq t(0)$ let $t_{j} \in B$ be such that

$$
\left.t_{j} \sqsubseteq\langle t(0)-j, t(0)-j+1, \ldots, t(0)-1)\right\rangle^{\wedge} t .
$$

Therefore $t_{0}=t$ and for every $j<t(0) t_{j+1} \triangleleft t_{j}$. We have

$$
f(t)(0) \geq f\left(t_{1}\right)(0)+1 \geq \cdots \geq f\left(t_{t(0)}\right)(0)+t(0) \geq t(0) .
$$

Now suppose $i+1<\operatorname{lh}(f(t))$ and choose any $t^{\prime} \in B$ such that $t \triangleleft t^{\prime}$ : we have $f(t) \triangleleft f\left(t^{\prime}\right)$ and, since $C$ is smooth, $i<\operatorname{lh}\left(f\left(t^{\prime}\right)\right)$. By the induction hypothesis $f(t)(i+1)=f\left(t^{\prime}\right)(i) \geq t^{\prime}(i)=t(i+1)$.

Lemma 3.4. If $B$ is a barrier, $B^{\prime}$ a smooth barrier and o.t. $(B)<0 . t .\left(B^{\prime}\right)$ then there exists no map $f: B \rightarrow B^{\prime}$ which preserves $\triangleleft$.

Proof. Suppose $f: B \rightarrow B^{\prime}$ preserves $\triangleleft$ and pick, by Lemma 3.2, $s \in B$ and $s^{\prime} \in B^{\prime}$ such that $s \subset s^{\prime}$. By Lemma $3.3 \ln (f(s)) \leq \operatorname{lh}(s)$ and $\forall i<$ $\operatorname{lh}(f(s)) s(i) \leq f(s)(i)$. Therefore $\ln (f(s))<\operatorname{lh}\left(s^{\prime}\right)$ and $\forall i<\ln (f(s)) s^{\prime}(i) \leq$ $f(s)(i)$. This contradicts the smoothness of $B^{\prime}$. 
In [15] it was claimed that every barrier of indecomposable order type does not contain a subbarrier of smaller order type: this was shown to be false in [1], but it is true if we restrict ourselves to smooth barriers, as the following corollary shows.

Corollary 3.5. (1) Every smooth barrier does not contain a subbarrier of smaller order type.

(2) Every smooth barrier has indecomposable order type.

Proof. (1) Let $B$ be a smooth barrier and $B^{\prime}$ be a subbarrier of $B$. The identity map from $B^{\prime}$ into $B$ obviously preserves $\triangleleft$. By Lemma 3.4 o.t. $\left(B^{\prime}\right)=$ o.t. $(B)$.

(2) follows from (1) because every barrier with nonindecomposable order type contains a subbarrier of smaller order type.

Theorem 3.6. For any countable indecomposable ordinal $\alpha$ there exists a partial ordering which is not $\alpha$-wqo but is $\beta$-wqo for every $\beta<\alpha$. In particular, for every countable $\alpha$ there exists a partial ordering which is $\alpha$-wqo but not bqo.

Proof. By Theorem 1.8 it suffices to find a reflexive binary relation which is not an $\alpha$-well relation but is a $\beta$-well relation for any $\beta<\alpha$.

By Lemma 3.1 let $B^{\prime}$ be a smooth barrier such that o.t. $\left(B^{\prime}\right)=\alpha$. Let $R$ be the relation on $B^{\prime}$ defined by $s R t \longleftrightarrow s \notin t$. The identity map from $B^{\prime}$ into itself shows that $R$ is not an $\alpha$-well relation. If $B$ is a barrier such that o. t. $(B)<\alpha$ Lemma 3.4 ensures that there is no map from $B$ into $B^{\prime}$ which is bad with respect to $R$.

Corollary 3.7. For any countable indecomposable ordinal $\alpha$ there exists a partial ordering $Q$ such that $Q^{<\alpha}$ is not wqo but $Q^{<\beta}$ is wqo for any indecomposable $\beta<\alpha$. In particular, for every countable $\alpha$ there exists a partial ordering such that $Q^{<\alpha}$ is wqo but $\tilde{Q}$ is not wqo.

Proof. Immediate from Theorems 2.8 and 3.6.

\section{Alternative Definitions OF $\alpha$-WQO}

Corollary 3.5 shows that smooth barriers have some nice properties that other barriers may not satisfy. It is natural to ask whether there are "enough" smooth barriers so that the definition of $\alpha$-wqo (and hence also of bqo) can be modified to deal only with maps with domain a smooth barrier. This is indeed the case and will be proved in this section. We will also give another definition of $\alpha$-wqo by measuring barriers and blocks not by their order type but by their height.

Even if we will not state this explicitly all these arguments apply also to the definition of $\alpha$-well relation.

Definition. If $B \subseteq[\mathbb{N}]^{<\omega}$ is such that two elements of $B$ are incomparable under $\subset$ let

$$
\begin{aligned}
T(B) & =\left\{s \in[\operatorname{base}(B)]^{<\omega} \mid \forall i<\operatorname{lh}(s) s[i] \notin B\right\}, \\
T^{*}(B) & =\left\{s \in[\operatorname{base}(B)]^{<\omega} \mid \exists t \in T(B) t \lll s\right\},
\end{aligned}
$$

where $t \ll s$ means that $\operatorname{lh}(t)=\operatorname{lh}(s)$ and $\forall i<\operatorname{lh}(s) t(i) \leq s(i)$. Both $T(B)$ and $T^{*}(B)$ are trees and we define $B^{*}=L_{T \cdot(B)}$.

Notice that $L_{T(B)}=B$ and $T\left(B^{*}\right)=T^{*}(B)$. 
Lemma 4.1. $B^{*}$ is smooth.

Proof. Suppose that $s, s^{\prime} \in B^{*}$ are such that $\operatorname{lh}(s)<\operatorname{lh}\left(s^{\prime}\right)$ and $\forall i<\operatorname{lh}(s) s^{\prime}(i)$ $\leq s(i)$. Since $s^{\prime} \in T^{*}(B)$ there exists $t^{\prime} \in T(B)$ such that $t^{\prime} \ll s^{\prime}$. If $t=$ $t^{\prime}[\operatorname{lh}(s)+1]$ we have $t \in T(B)$ and $\left.\left.t \ll s\right\} s^{\prime}(\ln (s))\right\rangle$, that is $\left.\left.s\right\} s^{\prime}(\ln (s))\right\rangle \in$ $T^{*}(B)$. This contradicts $s \in L_{T^{*}(B)}=B^{*}$.

Definition. If $B$ is a block then $T(B)$ is a well-founded tree and we define the height of $B$ by letting $\mathrm{ht}(B)=\mathrm{ht}(T(B))$.

Lemma 4.2. If $B$ is a block then the following hold:

(1) $T^{*}(B)$ is a well-founded tree.

(2) $B^{*}$ is a smooth barrier.

(3) $\operatorname{ht}\left(B^{*}\right)=\operatorname{ht}(B)$.

Proof. (1) Suppose $T^{*}(B)$ is not well-founded and let $X \in[\text { base }(B)]^{\omega}$ be such that $\forall i X[i] \in T^{*}(B)$. Then for every $i$ there exists $t \in T(B)$ such that $t \ll X[i]$. Hence the tree $\{t \in T(B) \mid t \lll X[\ln (t)]\}$ is an infinite finitely branching tree and by König's lemma is not well-founded. A fortiori $T(B)$ is not well-founded, against $B$ being a block.

(2) By Lemma 4.1 to prove that $B^{*}$ is a smooth barrier it suffices to show that it is a block. It is clear that two elements of $B^{*}$ are incomparable under $ᄃ$. Let $X \in[\operatorname{base}(B)]^{\omega}$ : by (1) there exists $k$ such that $X[k] \in T^{*}(B)$ and $X[k+1] \notin$ $T^{*}(B)$. To complete the proof it suffices to show that $X[k] \in B^{*}=L_{T^{*}(B)}$, that is that for every $n$ we have $X[k]\langle n\rangle \notin T^{*}(B)$. If $X[k]\langle n\rangle \in T^{*}(B)$ there exists $t\langle m\rangle \in T(B)$ such that $t \ll X[k]$ and $m \leq n$. Therefore $t \notin B$ and hence $t \nmid X(k)\rangle \in T(B)$. But $t \gamma X(k)\rangle \ll X[k] \chi X(k)\rangle=X[k+1]$ and therefore $X[k+1] \in T^{*}(B)$, a contradiction.

(3) By induction on $T^{*}(B)$ (which is well-founded by (1)) it is easy to prove that for all $s \in T^{*}(B)$ we have $h_{T^{*}(B)}(s)=\max \left\{h_{T(B)}(t) \mid t \in T(B) \wedge t \ll s\right\}$ (this uses the fact that for every $s \in T^{*}(B)$ the set $\{t \in T(B) \mid t \ll s\}$ is finite). Therefore $\operatorname{ht}\left(T^{*}(B)\right)=\operatorname{ht}(T(B))$ and hence $\operatorname{ht}\left(B^{*}\right)=\operatorname{ht}(B)$.

Definition. Let $\alpha$ be a countable ordinal and $Q$ a quasi-ordering. $Q$ is smooth$l y \alpha$-wgo if for every smooth barrier $B$ with o.t. $(B) \leq \alpha$ every map from $B$ into $Q$ is good. $Q$ is height- $\alpha$-wqo if for every block $B$ with $\operatorname{ht}(B) \leq \alpha$ every map from $B$ into $Q$ is good.

Theorem 4.3. Let $\alpha$ be a countable ordinal and $Q$ a quasi-ordering. Then the following are equivalent:

(1) $Q$ is $\omega^{\alpha}$-wqo.

(2) $Q$ is smoothly $\omega^{\alpha}$-wqo.

(3) $Q$ is height- $\alpha$-wqo.

(4) $Q^{<\omega^{\alpha}}$ is wqo.

Proof. It is obvious that (1) implies (2) while (4) implies (1) follows from Theorem 2.8 .

To show that (2) implies (3) suppose $f: B \rightarrow Q$ is bad where $B$ is a block with $h t(B) \leq \alpha$. By Lemma 4.2(3) we have $h t\left(T^{*}(B)\right) \leq \alpha$ and hence, by Lemma 2.3 , o.t. $\left(B^{*}\right) \leq \omega^{\alpha}$. Moreover, by Lemma $4.2(2), B^{*}$ is a smooth barrier. Define $f^{*}: B^{*} \rightarrow Q$ by $f^{*}(s)=f(t)$ where $t$ is the unique element of $B$ such that $t \sqsubseteq s . f^{*}$ is bad and shows that $Q$ is not smoothly $\omega^{\alpha}$-wqo. 
In $\S 2$ we proved that (1) implies (4). To prove that (3) implies (4) slight modifications of a couple of those proofs are sufficient: Lemma 2.1 needs an application of Lemma 1.2 to start with, while in the proof of Lemma $2.4 B$ is precisely a block with $\mathrm{ht}(B) \leq \alpha$ (if we begin with a bad sequence in $\mathscr{H}_{<\omega^{a}}$ ). The remaining parts of $\S 2$ apply also if the original hypothesis is (3) in place of (1).

Corollary 4.4. Let $Q$ be a quasi-ordering. Then the following are equivalent:

(1) $Q$ is bqo.

(2) Every map from a smooth barrier into $Q$ is good.

Proof. Immediate from Theorem 4.3.

\section{BQO IS COMPLETE $\Pi_{2}^{1}$}

In this section we study the complexity of the notion of better-quasi-ordering using techniques and concepts from recursion theory and descriptive set theory and in particular we establish the position of bqo in the analytical hierarchy. For background on these subjects see [18] and [11].

Definition. Let $\mathscr{B}=\{e \mid e$ is an index for a recursive bqo $\}$ and $B=\left\{Q \in 2^{\omega} \mid\right.$ $Q$ is a code for a bqo on $\mathbb{N}$.

An immediate Tarski-Kuratowski computation shows that $\mathscr{B}$ is a $\Pi_{2}^{1}$ subset of $N$ and $B$ is a (lightface) $\Pi_{2}^{1}$ subset of the Cantor space $2^{\omega}$. We will show that $\mathscr{B}$ is complete $\Pi_{2}^{1}$, i.e., that for every $\Pi_{2}^{1}$ set $P \subseteq N$ there exists a primitive recursive function $f: \mathbb{N} \rightarrow \mathbb{N}$ such that $\forall n(n \in P \longleftrightarrow f(n) \in \mathscr{B})$. This implies that $\mathscr{B}$ is not $\Sigma_{2}^{1}$. We will also show that $B$ is complete $\Pi_{2}^{1}$, i.e., that for every $\Pi_{2}^{1}$ set $\mathbb{P} \subseteq \omega^{\omega}$ there exists a recursive functional (i.e., a continuous function with a recursive code) $F: \omega^{\omega} \rightarrow 2^{\omega}$ such that $\forall X(X \in$ $\mathbf{P} \longleftrightarrow F(X) \in \mathbf{B})$. This implies that $\mathbf{B}$ is not $\Sigma_{2}^{1}$ and also that $B$ is a complete (boldface) $\Pi_{2}^{1}$ set. This latest completeness means that the previous property holds for all (not necessarily recursively coded) $\Pi_{2}^{1}$ subsets of $\omega^{\omega}$ if we allow $F$ to be an arbitrary continuous function.

All these results will follow from the following lemma.

Lemma 5.1. Let $\mathbb{P} \subset \omega^{\omega}$ be a $\Pi_{2}^{1}$ set. For each $g \in \omega^{\omega}$ we can define, primitive recursively in $g$, a reflexive binary relation $R_{g}$ on a countable set $Q_{g}$ such that $g \in \mathbb{P}$ if and only if $\boldsymbol{R}_{\boldsymbol{g}}$ is a better relation. Moreover there is a primitive recursive functional taking $g$ into $\left(Q_{g}, R_{g}\right)$.

Proof. By the Kleene normal form theorem (using the fact that $[\mathbb{N}]^{\omega}$ is isomorphic to $\left.\omega^{\omega}\right)$ there exists a recursive set $C \subset \bigcup_{n} \omega^{n} \times 2^{n} \times[N]^{n}$ such that

$$
g \in \mathbb{P} \longleftrightarrow \forall f \in 2^{\omega} \exists X \in[\mathbb{N}]^{\omega} \forall n(g[n], f[n], X[n]) \notin C .
$$

We suppose that $(\langle\rangle,\langle\rangle,\langle\rangle) \notin C$ (otherwise $\mathrm{P}=\varnothing$ and for every $g$ we can take $R_{g}$ to be a fixed primitive recursive non-better relation). Moreover we can suppose that $C$ consists of sequences incomparable under $\sqsubset$.

Throughout this proof $\sigma$ and $\sigma^{\prime}$ will denote sequences in $2^{<\omega}$, while $s$ and $s^{\prime}$ will denote elements of $[\mathbb{N}]^{<\omega}$.

Let $g \in \omega^{\omega}$ be fixed and define $C_{g}=\{(\sigma, s) \mid(g[\ln (\sigma)], \sigma, s) \in C\}$ and

$$
C_{g}^{*}=\left\{(\sigma, s) \mid \forall t \lll s \exists i \leq \ln (s)(\sigma[i], t[i]) \in C_{g}\right\} .
$$


Notice that $C_{g}^{*}$ is primitive recursive in $g$. For any $f \in 2^{\omega}$ let $C_{g, f}=\{s \mid$ $\left.(f[\operatorname{lh}(s)], s) \in C_{g}\right\}$. Notice that the elements of $C_{g, f}$ are incomparable under $ᄃ$ and, using the constructions of $\S 4$, define $C_{g}^{*}, f$. Let $Q_{g}=\left\{(\sigma, s) \in C_{g}^{*} \mid\right.$ $\left.\forall i<\operatorname{lh}(s)(\sigma[i], s[i]) \notin C_{g}^{*}\right\}$ and define a binary relation $R_{g}$ on $Q_{g}$ by

$$
(\sigma, s) R_{\boldsymbol{g}}\left(\sigma^{\prime}, s^{\prime}\right) \longleftrightarrow \sigma \square \sigma^{\prime} \vee s \not s^{\prime}
$$

Notice that $Q_{g}$ and $R_{g}$ are primitive recursive in $g$ and that by the uniformity of the construction there is a primitive recursive functional taking $g$ into $\left(Q_{g}, R_{g}\right)$.

To complete the proof of the lemma by showing that $g \in \mathbb{P}$ if and only if $\boldsymbol{R}_{\boldsymbol{g}}$ is a better relation we need the following sublemma.

Sublemma. For any $f$ and $s$ we have that $s \in C_{\mathcal{g}, f}^{*}$ if and only if $(f[\ln (s)], s) \in$ $Q_{g}$.

Proof. $(\Rightarrow)$ Suppose that $s \in C_{g, f}^{*}=L_{T^{*}\left(C_{g, f}\right)}$ : for every $n s^{\wedge}\langle n\rangle \notin T^{*}\left(C_{g, f}\right)$ and we have that if $t \lll s$, for every $n t \gamma n\rangle \notin T\left(C_{g, f}\right)$. This implies that there exists $i \leq \ln (s)$ such that $t[i] \in C_{g, f}$ and therefore $(f[i], t[i]) \in C_{g}$. Hence $(f[\operatorname{lh}(s)], s) \in C_{g}^{*}$.

Moreover there exists $t \in T\left(C_{g, f}\right)$ such that $t \ll s$. Therefore for every $i<$ $\operatorname{lh}(s)$ we have $t[i] \notin C_{g, f}$, that is $(f[i], t[i]) \notin C_{g}$ and hence $(f[i], s[i]) \notin$ $C_{g}^{*}$. This implies that $(f[\operatorname{lh}(s)], s) \in Q_{g}$.

$(\Leftrightarrow)$ If $(f[\operatorname{lh}(s)], s) \in C_{g}^{*}$ and $\forall i<\operatorname{lh}(s)(f[i], s[i]) \notin C_{g}^{*}$ then for every $t \ll s$ there exists $i \leq \operatorname{lh}(s)$ such that $(f[i], t[i]) \in C_{g}$ and for at least one $t$ this $i$ is $\operatorname{lh}(s)$. Hence $\forall t \ll s \exists i \leq \operatorname{lh}(s) t[i] \in C_{g, f}$ and $\exists t \ll s t \in C_{g, f}$. Therefore $s \in T^{*}\left(C_{g, f}\right)$ and for every $n s\{n\rangle \notin T^{*}\left(C_{g, f}\right)$. Thus $s \in L_{T^{*}\left(C_{g, f}\right)}=$ $C_{g, f}^{*}$.

First suppose $g \in \mathbb{P}$ but $R_{g}$ is not a better relation: there exist a barrier $B$ with base $(B)=\mathbb{N}$ and a map $F: B \rightarrow Q_{g}$ which is bad with respect to $R_{g}$. Let us write $F(t)=(G(t), H(t))$. The badness of $F$ means that whenever $t, t^{\prime} \in B$ are such that $t \triangleleft t^{\prime}$ we have (1) $G(t) \sqsubseteq G\left(t^{\prime}\right)$ and (2) $H(t) \triangleleft H\left(t^{\prime}\right)$. From (1) it follows that for some $f \in 2^{\omega}$ we have $\forall t \in B \quad G(t) \subset f$ (this is because, if $\triangleleft^{*}$ is the transitive closure of $\triangleleft$, for any $t, t^{\prime} \in B$ there exists $u \in B$ such that $t \triangleleft^{*} u$ and $\left.t^{\prime} \triangleleft^{*} u\right)$. By the sublemma $\operatorname{mg}(H) \subseteq C_{g, f}^{*}$. Since $g \in \mathbb{P}$ there exists $X \in[\mathbb{N}]^{\omega}$ such that $\forall n(f[n], X[n]) \notin C_{g}$. If $t \in B$ is such that $t \subset X$, since $H$ preserves $\triangleleft$ and $C_{g}^{*}, f$ is smooth by Lemma 4.1, Lemma 3.3(2) applies and $\forall i<\operatorname{lh}(H(t)) X(i) \leq H(t)(i)$ : it is then easy to see that $F(t)=(f[\operatorname{lh}(H(t))], H(t)) \notin C_{g}^{*}$, a contradiction.

Now assume $g \notin \mathbb{P}$ : for some $f \in 2^{\omega}$ we have $\forall X \in[\mathbb{N}]^{\omega} \exists n X[n] \in$ $C_{g, f}$. Therefore $C_{g, f}$ is a block and hence, by Lemma 4.2(2), $C_{g, f}^{*}$ is a smooth barrier. Define $F: C_{g, f}^{*} \rightarrow Q_{g}$ by $F(s)=(f[\ln (s)], s)$. It is immediate to check that $F$ is bad with respect to $R_{g}$ and hence $R_{g}$ is not a better relation.

Theorem 5.2. $\mathscr{B}$ is complete $\Pi_{2}^{1}$.

Proof. For every $n \in \mathbb{N}$ let $g_{n} \in \omega^{\omega}$ be the function such that for every $i$ $g_{n}(i)=n$. Let $P \subseteq \mathbb{N}$ be a $\Pi_{2}^{1}$ set: define $\mathbb{P} \subseteq \omega^{\omega}$ so that $\forall n(n \in P \longleftrightarrow$ $\left.g_{n} \in \mathbb{P}\right)$. This can be done in such a way that $\mathbb{P}$ is $\Pi_{2}^{1}$. For every $n \in \mathbb{N}$ let $R_{g_{n}}$ be the reflexive binary relation given by Lemma 5.1 and let $\preceq_{n} \subseteq R_{g_{n}}$ be 
the partial ordering given by Corollary 1.9. Then $\preceq_{n}$ is uniformly primitive recursive in $g_{n}$ and there is a primitive recursive function $f$ such that $f(n)$ is an index for $\preceq_{n}$. Then $\forall n \in \mathbb{N}(n \in P \longleftrightarrow f(n) \in \mathscr{B})$ and the proof is complete.

Theorem 5.3. B is complete $\Pi_{2}^{1}$.

Proof. Immediate from Lemma 5.1 and Corollary 1.9.

\section{REFERENCES}

1. M. Assous, Caracterisation du type d'ordre des barrières de Nash-Williams, Publ. Dep. Math. Nouvell Sér. 11 (1974), 89-106.

2. P. Clote, A recursion theoretic analysis of the clopen Ramsey theorem, J. Symbolic Logic 49 (1984), 376-400.

3. - The metamathematics of Fraisse's order type conjecture, Recursion Theory Week (K. Ambos-Spies, G. H. Müller, and G. E. Sacks, eds.), Lecture Notes in Math., vol. 1432, Springer-Verlag,, Berlin and New York, 1990, pp. 41-56.

4. R. Fraissé, Theory of relations, North-Holland, Amsterdam, 1986.

5. J. B. Kruskal, The theory of well-quasi-ordering: a frequently discovered concept, J. Combin. Theory Ser. A 13 (1972), 297-305.

6. R. Laver, On Fraisse's order type conjecture, Ann. of Math. (2) 93 (1971), 89-111.

7. A. Louveau and J. Saint-Raymond, On the quasi-ordering of Borel linear orders under embeddability, J. Symbolic Logic 55 (1990), 537-560.

8. R. Mansfield and G. Weitkamp, Recursive aspects of descriptive set theory, Oxford Univ. Press, New York, 1985.

9. A. Marcone, Foundations of bqo theory and subsystems of second order arithmetic, Ph.D. thesis, The Pennsylvania State Univ., 1993.

10. E. C. Milner, Basic wqo- and bqo-theory, Graphs and Orders (I. Rival, ed.), Reidel, Boston, Mass., 1985, pp. 487-502.

11. Y. N. Moschovakis, Descriptive set theory, North-Holland, Amsterdam, 1980.

12. C. St. J. A. Nash-Williams, On well-quasi-ordering tranfinite sequences, Proc. Cambridge Philos. Soc. 61 (1965), 33-39.

13. _ On well-quasi-ordering infinite trees, Proc. Cambridge Philos. Soc. 61 (1965), 697720.

14. 273-290.

15. M. Pouzet, Sur les prémeilleurordres, Ann. Inst. Fourier (Grenoble) 22 (1972), 1-20.

16. __ Applications of well quasi-ordering and better quasi-ordering, Graphs and Orders (I. Rival, ed.), Reidel, Boston, Mass., 1985, pp. 503-519.

17. __ Graphs and posets with no infinite independent set, Finite and Infinite Combinatorics in Sets and Logic, (N. W. Sauer et al., eds.), Kluwer, 1993, pp. 313-335.

18. H. Rogers, Theory of recursive functions and effective computability, McGraw-Hill, New York, 1967.

19. J. G. Rosenstein, Linear orderings, Academic Press, New York, 1982.

20. S. Shelah, Better quasi-orders for uncountable cardinals, Israel J. Math. 42 (1982), 177-226.

21. R. A. Shore, On the strength of Fraisse's conjecture, Logical Methods: In Honor of Anil Nerode's 60th Birthday (J. N. Crossley, J. B. Remmel, M. E. Sweedler, R. A. Shore, eds.), Birkhäuser, Cambridge, Mass., 1993, pp. 782-813. 
22. S. G. Simpson, Bgo-theory and Fraisse's conjecture, [8], pp. 124-138.

23. , Subsystems of $Z_{2}$ and reverse mathematics, appendix to G. Takeuti, Proof Theory, 2nd ed., North-Holland, Amsterdam, 1986.

Departmant of Mathematics, The Pennsylvania State University, University Park, Pennsyivania 16802

Current address: Dipartimento di Matematica, Universita di Torino, via Carlo Alberto 10, 10123 Torino, Italy

E-mail address: marconeldm.unito.it 